\title{
Reflets
}

Revue d'intervention sociale et communautaire

\section{Analyse intersectionnelle des défis et enjeux des inégalités sociales sur le marché du travail : l'expérience de vingt femmes congolaises résidant au Québec et en Ontario An Intersectional Analysis of Issues and Challenges Concerning Social Inequalities in the Workforce: the Case of Twenty Congolese Women in Quebec and Ontario}

\author{
Nsimire Namululi, Rachid Bagaoui et Komi Hemedzo
}

Volume 24, numéro 1, printemps 2018

Les inégalités sociales en contexte minoritaire

URI : https://id.erudit.org/iderudit/1051522ar

DOI : https://doi.org/10.7202/1051522ar

\section{Aller au sommaire du numéro}

\section{Éditeur(s)}

Reflets, Revue d'intervention sociale et communautaire

ISSN

1203-4576 (imprimé)

1712-8498 (numérique)

Découvrir la revue

Citer cet article

Namululi, N., Bagaoui, R. \& Hemedzo, K. (2018). Analyse intersectionnelle des défis et enjeux des inégalités sociales sur le marché du travail : l'expérience de vingt femmes congolaises résidant au Québec et en Ontario. Reflets, 24(1),

98-126. https://doi.org/10.7202/1051522ar

\section{Résumé de l'article}

Dans une perspective intersectionnelle qui articule les différents marqueurs identitaires, notamment la couleur de la peau, le sexe, l'origine ethnique, la taille, la langue et la religion, cet article est basé sur une étude qui veut savoir dans quelle mesure ces variables prédisposent les femmes noires originaires de l'Afrique aux discriminations, mythes, racisme, préjugés et stéréotypes sur le marché de l'emploi. L'article présente les résultats d'une analyse qualitative qui se concentre sur les expériences de femmes immigrantes congolaises sur le marché de l'emploi en Ontario et au Québec, deux grandes provinces du Canada. Les résultats soulèvent trois variables qui illustrent les difficultés de ces femmes sur le marché de l'emploi : la couleur de la peau, le sexe et l'origine ethnique. L'article montre que ces femmes sont discriminées

systématiquement, qu'elles soient nées au Canada ou qu'elles y aient immigré.
Tous droits réservés (C) Reflets, Revue d'intervention sociale et communautaire, 2018
Ce document est protégé par la loi sur le droit d'auteur. L'utilisation des services d’Érudit (y compris la reproduction) est assujettie à sa politique d'utilisation que vous pouvez consulter en ligne. 


\title{
Analyse intersectionnelle des défis et enjeux des inégalités sociales sur le marché du travail : l'expérience de vingt femmes congolaises résidant au Québec et en Ontario
}

\author{
Nsimire Namululi \\ Doctorante en sociologie, Université Laurentienne
}

Rachid Bagaoui, Ph. D.

Professeur agrégé, Département de sociologie, Université Laurentienne

Komi Hemedzo, Ph. D.

Professeur associé, Département de sociologie, Université Laurentienne

\section{Résumé}

Dans une perspective intersectionnelle qui articule les différents marqueurs identitaires, notamment la couleur de la peau, le sexe, l'origine ethnique, la taille, la langue et la religion, cet article est basé sur une étude qui veut savoir dans quelle mesure ces variables prédisposent les femmes noires originaires de l'Afrique aux discriminations, mythes, racisme, préjugés et stéréotypes sur le marché de l'emploi. L'article présente les résultats d'une analyse qualitative qui se concentre sur les expériences de femmes immigrantes congolaises sur le marché de l'emploi en Ontario et au Québec, deux grandes provinces du Canada. Les résultats soulèvent trois variables qui illustrent les difficultés de ces femmes sur le marché de l'emploi : la couleur de la peau, le sexe et l'origine ethnique. L'article montre que ces femmes sont discriminées systématiquement, qu'elles soient nées au Canada ou qu'elles y aient immigré.

Mots clés : immigrante, discrimination, sexisme, résilience, emploi, mythe, obstacle, combat, intégration, racisme 


\section{An Intersectional Analysis of Issues and Challenges Concerning Social Inequalities in the Workforce: the Case of Twenty Congolese Women in Quebec and Ontario}

\section{Abstract}

In an intersectional perspective that articulates the different identity markers such as skin colour, sex, ethnic origin, size, language and religion, this article is based on a study of the extent to which these variables predispose black women originating from Africa to discriminations, myths, racism, prejudices and stereotypes in the labor market. The article presents the results of a qualitative analysis that focuses on the experiences of Congolese immigrant women in the labor market in Ontario and Quebec, two major provinces of Canada. The results raise three variables that illustrate the difficulties these women face in the job market. These are the colour of the skin, gender and ethnicity. The article shows that these women are systematically discriminated against, whether they were born in Canada or they immigrated to Canada.

Key words: immigrant, discrimination, sexism, resilience, employment, myth, obstacle, fight, integration, racism

\section{Introduction}

Les questions des inégalités sur le marché du travail touchent plusieurs couches de la société et communautés vivant au Canada. C'est le cas notamment des femmes immigrantes. Certes, les femmes ne sont pas les seules à rencontrer des obstacles sur le marché du travail. Les hommes sont aussi pénalisés, comme le montrent depuis longtemps plusieurs études (Picot et Sweetman, 2005; Gilmore, 2008; Schellenberg et Maheux, 2007). Malgré les progrès et les réformes contre les discriminations, les personnes de

"Malgré les progrès et les réformes contre les discriminations, les personnes de différents groupes ethniques continuent de souffrir des injustices et des inégalités... " différents groupes ethniques continuent de souffrir des injustices et des inégalités qui persistent. Les facteurs qui expliquent les difficultés que rencontrent les immigrantes et immigrants pour intégrer le marché de l'emploi sont nombreux. Soulignons entre autres la nonreconnaissance des diplômes étrangers, qui sont soit ignorés par les employeurs pour les emplois qualifiés, 
soit considérés comme contre-productifs dans les emplois peu qualifiés (Chicha, 2009). Il faut évoquer également l'obstacle que peut représenter l'absence d'une expérience professionnelle, très souvent présentée comme un obstacle incontournable, sans que les employeurs puissent préciser ce qu'elle représente vraiment pour eux. Les écarts au niveau des salaires et de l'emploi constituent un autre critère d'inégalité sur le marché du travail (Nikuze, 2011). Le poids des préjugés chez les employeurs est aussi un facteur déterminant qui contribue à la discrimination que connaissent les femmes immigrantes. Les employeurs ont tendance à choisir ou à rechercher inconsciemment des personnes qui leur ressemblent ou parfois même à éviter certains groupes évalués négativement, parce que socialement stigmatisés (Eid, 2012).

Les recherches qui ont analysé les discriminations vécues par les personnes immigrantes cherchant à intégrer le marché de l'emploi montrent que les caractéristiques corporelles, comme les traits physiques et la couleur de la peau, contribuent à freiner leur accès au travail (Mianda, 1998; Bals, 1999; Arsenault, 2003; Pierre, 2005; LY, 2007; Chicha, 2009; Lemoine, 2010; Nikuze, 2011; Eid, 2012; Hyppolite, 2012; Imboua, 2012). Ainsi, certaines caractéristiques corporelles différentes de celles de la majorité continuent de poser de sérieux problèmes dans nos sociétés qui exigent de plus en plus des standards d'image corporelle parfaite et dictent les normes entre ce qui est beau et ce qui est laid. Bref, le monde du travail ne cesse d'encourager les images stéréotypées sur le corps des femmes (Naves, 2016). Les femmes noires se retrouvent aussi prises dans cette catégorie de beauté en étant exclues. En effet, la couleur de la peau de ces femmes constitue un facteur de leur exclusion. Elle ne ferait pas partie des caractéristiques de beauté dans notre société. Ladite société les discrimine toujours sur la base des références qui ne sont pas les leurs.

La question principale de notre recherche était de comprendre comment les femmes

"...comment les femmes con-

golaises vivent ces inégalités..." congolaises vivent ces inégalités sur le marché du travail et quelle lecture elles font de leur situation. Par ailleurs, dans le présent article, notre objectif n'est pas tant de confronter nos observations avec la littérature, mais plutôt d'entendre l'opinion des femmes immigrantes d'origine congolaise sur les difficultés qu'elles rencontrent sur le marché du travail, comment elles vivent ces expériences et comment elles expliquent leurs propres difficultés et donc les inégalités qu'elles subissent.

La problématique de cet article est bien entendu d'appréhender les inégalités dont seraient victimes les femmes immigrées au Québec et en Ontario à travers les concepts de l'inégalité, de l'intersectionnalité et de la femme racisée. Il faut donc éviter des écueils de subjectivité qui pourraient prêter le flanc aux critiques faciles, allant de reproches de 
militantisme et d'ambiguïté à la victimisation en passant par l'excès d'émotivité, tous incompatibles la plupart du temps avec la science. Même si Yuval-Davis (2006) et d'autres tenants de l'approche intersectionnelle ont largement démontré que celle-ci dispose d'un statut pour donner corps aux études féministes, la question qui demeure fondamentale est de trouver des moyens de nous approprier ce statut sur le plan de la méthodologie sociologique, afin d'aborder de façon rigoureuse et méthodique la question des inégalités dont seraient victimes les femmes africaines noires immigrées au Canada. Tels sont les grands défis auxquels répond cet article.

\section{Cadre théorique}

L'expérience des femmes congolaises renvoie à leur réalité à la fois de femmes (genre), "Pour rendre compte de la complexité de l'expérience des femmes congolaises, la recherche a utilisé l'approche de l'intersectionnalité des facteurs d'inégalité et de discrimination..." immigrantes (originale sociale) et racisées. Pour rendre compte de la complexité de l'expérience des femmes congolaises, la recherche a utilisé l'approche de l'intersectionnalité des facteurs d'inégalité et de discrimination, qui sont nombreux dans les recherches en sciences sociales. Ils sont observables par le biais de variables comme l'inégalité liée au sexe ou à la couleur de la peau. Cependant, ces différents types d'inégalité sont concentrés dans ce qu'il convient d'appeler les facteurs d'inégalité et de discrimination face à l'emploi surtout pour les personnes immigrantes.

Bilge (2009) note que l'intersectionnalité renvoie à une théorie transdisciplinaire visant à appréhender la complexité des identités et des inégalités sociales par une approche intégrée. L'approche de l'intersectionnalité offre une analyse intégrée dans la mesure où elle aborde presque tous les indicateurs des inégalités sociales en se refusant toute posture de simple dénonciation des inégalités à l'endroit des femmes. Concrètement, l'intersectionnalité peut être considérée comme une importante contribution théorique du féminisme (Herla, 2010). Crenshaw (2005), qui est à l'origine du concept de l'intersectionnalité, cherchait à comprendre la variété des interactions entre les rapports de genre et de race vécues par les femmes afro-américaines. Pour Crenshaw, les inégalités subies par les femmes proviennent du rapport de force qu'on nomme le pouvoir. L'intersectionnalité des rapports de pouvoir produit en effet des tensions, des conflits et des effets destructeurs et déstructurants dans les processus de mobilisation des mouvements sociaux (Dorlin, 2005). C'est pourquoi les théoriciennes doivent combiner dans leur 
analyse l'effet à la fois du genre et de la race et le rôle que ces marqueurs jouent dans les expériences des femmes (Crenshaw, 2005). Ce sont précisément ces dimensions que nous examinons à travers les indicateurs de l'inégalité des femmes noires immigrées face à l'emploi, leurs espoirs, leurs déceptions et les obstacles auxquels elles sont souvent confrontées dans leur quête de bien-être social par le truchement de la formation et de l'emploi. Selon Davis (2015), l'intersectionnalité offre une façon inattendue de surmonter ces incompatibilités entre théorie féministe de la race, de la classe et du genre, et théorie féministe postmoderne. La théorie de l'intersectionnalité est l'une des pierres angulaires de cette étude, car, pour Davis (2015), non seulement elle reprend le projet politique de rendre visibles les conséquences sociales et matérielles des catégories de genre, de classe et de race, mais elle le fait à l'aide de méthodologies compatibles avec le projet poststructuraliste qui vise à déconstruire les catégories, à démasquer l'universalisme et à explorer le fonctionnement dynamique et contradictoire du pouvoir. Ainsi, toujours selon Davis (2015), l'intersectionnalité donne à la théorie féministe poststructuraliste une crédibilité politique, lui permettant de contrer certaines critiques issues du féminisme multiculturel, qui lui reprochent de s'être trop écartée des réalités matérielles de la vie des femmes et d'être trop relativiste pour servir leurs luttes politiques concrètes (Davis, 2015). Le défi de notre étude et, par ricochet, le défi de notre article est de taille, quand on sait que les analyses qui s'appuient sur l'intersectionnalité font l'objet de critiques acerbes. Ainsi, selon Corbeil et Marchand (2007), les premières critiques adressées au féminisme, considéré hégémonique et ethnocentrique, émanent principalement de femmes noires américaines qui rappelleront à quel point le racisme demeure, pour elles, une expérience aussi visible, quotidienne et virulente que le sexisme, sinon davantage pour certaines d'entre elles. Les initiatrices de ce discours que sont bell hooks (1981; 1984) et Angela Davis (1981) dénoncent également le fait que la réalité quotidienne des femmes racisées et les discriminations dont elles sont l'objet ne trouvent guère de résonance, ni dans le mouvement de libération des Noirs, ni au sein du mouvement d'émancipation des femmes. Ainsi, poser un regard scientifique sur le cas de certaines femmes congolaises vivant en Ontario et au Québec par le biais de l'intersectionnalité nécessite une méthodologie bien précise.

\section{Méthode de recherche}

Pour comprendre les expériences de recherche de travail des femmes congolaises au Canada ou les expériences de celles qui luttent pour garder leurs emplois, nous avons adopté 

de recherche de travail des femmes congolaises au Canada ou les expériences de celles qui luttent pour garder leurs emplois, nous avons adopté une approche qualitative."
"Pour comprendre les expériences

une approche qualitative. Nous avons recueilli des entretiens sur les perceptions et les explications que ces femmes donnent à leurs expériences de recherche d'emploi. L'analyse des expériences des femmes congolaises s'est concentrée sur les stratégies collectives et individuelles employées par ces dernières. L'échantillon a été formé à partir des critères suivants : être femme congolaise ou d'origine congolaise ayant un statut de résidente permanente ou ayant acquis la nationalité canadienne, avoir 18 ans ou plus, être salariée ou être en situation de demandeuse d'emploi. Les femmes devaient répondre à ce critère d'âge pour faire partie de l'échantillon, même si l'âge minimum pour travailler au Canada est fixé à 16 ans. Tous les entretiens ont été réalisés en face à face, et chacun a duré près d'une heure trente minutes. En d'autres termes, c'était la durée moyenne des vingt entretiens. À l'occasion, il a fallu aller sur place pour pouvoir rencontrer les femmes qui ont fait partie de l'échantillon.

Par souci de rigueur intellectuelle et scientifique, nous avons vérifié auprès des femmes interviewées si la transcription des entretiens était conforme à leur attente, afin de la corriger, au besoin. Cette étape franchie, toutes les voies étaient alors ouvertes afin de faire les analyses et les interprétations des entretiens, le regroupement des données en sous-catégories, l'encodage, le décryptage et l'interprétation des données. Enfin, pour des raisons de fiabilité méthodologique, un collègue externe a jugé la validité de tout le processus par le biais d'une méthode souvent qualifiée d'inter-juge. Le guide d'entretien a abordé deux grandes thématiques. La première concerne les obstacles que les interviewées ont rencontrés avant d'entrer sur le marché de l'emploi, et la seconde, les obstacles auxquels elles ont été confrontées alors qu'elles étaient déjà sur le marché de l'emploi. Avant le début de l'entretien proprement dit, les objectifs de la recherche ont été rappelés aux membres de l'échantillon, même si cela leur avait été présenté une première fois par téléphone ou par courriel. Au cours des entretiens, diverses questions ont été abordées concernant leur parcours, allant de la raison de leur migration à leur passé professionnel pour aboutir à leur condition actuelle. Nous avons évoqué aussi les rapports qu'elles entretiennent à la fois avec leur pays d'accueil et avec leur pays d'origine. Bien que l'analyse des résultats se concentre plus sur les expériences de recherche d'emploi des participantes au Canada, un guide a été ainsi élaboré en vue de recueillir le plus d'informations possibles sur leurs parcours avant et après la migration, afin de comprendre leurs expériences antérieures. Il vise aussi à saisir d'où elles viennent et comment elles se sont forgé un chemin sur 
le marché du travail au Canada. Il était important de faire ce retour sur leur passé, qui renferme entre autres leurs parcours éducatifs et professionnels, les conditions sociales et matérielles de leurs familles ainsi que les motifs qui les avaient obligées à quitter leur pays d'origine.

"Au total, vingt femmes résidant dans deux provinces canadiennes (soit l'Ontario et le Québec) se sont portées volontaires pour la recherche."

Au total, vingt femmes résidant dans deux provinces canadiennes (soit l'Ontario et le Québec) se sont portées volontaires pour la recherche. L'échantillon est donc composé de quatre résidentes de Sudbury, dix de Montréal, trois de Toronto et trois d'Ottawa. L'âge de ces femmes varie entre vingt et cinquante-sept ans. Dix-huit femmes sont des immigrantes en provenance du Congo, alors que deux d'entre elles sont nées au Canada. Ces dernières ont jugé utile de participer à la recherche, car elles se sentent concernées et affectées par les effets du racisme et des discriminations, au même titre que les femmes congolaises immigrantes. Deux autres participantes ont immigré au Canada alors qu'elles étaient très jeunes. Quant à celles qui ne sont pas nées au Canada, elles ont immigré entre 1987 et 2011. La majorité de ces femmes ont obtenu la citoyenneté canadienne, sauf trois participantes qui ont encore le statut de résidentes permanentes. La majorité de ces femmes ont immigré au Canada, en raison de guerres qui prévalaient dans leur pays d'origine.

\section{Analyse des résultats}

L'expérience des femmes congolaises peut se lire par rapport aux différents obstacles que les participantes ont dû affronter dans leur parcours professionnel. Cette expérience peut être scindée en deux, soit les obstacles pour intégrer le marché du travail et les obstacles rencontrés en cours de l'emploi. Comme l'indiquent les données de l'Institut canadien de recherches sur les femmes :

Peu importe leur niveau de scolarité, les femmes immigrantes gagnent moins que les femmes nées au Canada. Les immigrantes ont moins souvent un emploi que les immigrants et les femmes non immigrantes, même quand elles ont un diplôme universitaire. (ICREF, 2003, p. 5-6)

L'Institut canadien de recherches sur les femmes résume assez bien ces obstacles, et les femmes congolaises font face à de nombreux autres obstacles qui freinent leur insertion sur le marché de l'emploi. 


\section{Obstacles rencontrés par les femmes congolaises pour intégrer le marché de l'emploi}

"...ont fait état... du manque de reconnaissance des acquis et des expériences antérieures, du manque de références et d'expérience canadienne, de problèmes à concilier le travail et la famille etlou les études, etc."
Les participantes ont évoqué différents obstacles qu'elles ont rencontrés avant d'intégrer le marché du travail au Canada. Elles ont fait état, entre autres, du manque de reconnaissance des acquis et des expériences antérieures, du manque de références et d'expérience canadienne, de problèmes à concilier le travail et la famille et/

ou les études, etc.

"La première chose qui m’avait choquée quand je suis allée demander de l'emploi, c'est qu'on m'avait demandé si j'avais de l'expérience de travail au Canada. Que je présente des références de mes anciens employeurs. Je venais d'arriver directement de mon pays. Où est-ce que l'employeur pensait que je devrais trouver cette expérience? Je le veux bien qu'on me donne du travail d'abord pour que j'aie de l'expérience canadienne pour ensuite présenter des références. Je trouve que c'est ridicule d'exiger aux immigrants les références sur leurs expériences de travail, alors qu'ils n'ont jamais travaillé au Canada. C'est vraiment de la discrimination envers les immigrants. » (Participante A3)

Tous ces facteurs nourrissent le sentiment de discrimination que vivent ces femmes.

"Au Canada, les femmes sont discriminées, mais, pour nous, les femmes noires, c'est pur. Notre couleur de peau noire nous trahit dans plusieurs circonstances, surtout quand on cherche le travail."
"Au Canada, les femmes sont discriminées, mais, pour nous, les femmes noires, c'est pur. Notre couleur de peau noire nous trahit dans plusieurs circonstances, surtout quand on cherche le travail. Elle nous dispose à plusieurs inégalités dans la société. " (Participante A 10)

Or, certaines étaient enseignantes, infirmières, entrepreneures, vendeuses, sagesfemmes, etc., des conditions qui, sans doute, leur permettaient de s'épanouir tant sur le plan personnel que sur le plan professionnel, tout en contribuant au bien-être de leurs familles. 


\section{Non-reconnaissance des diplômes et des expériences antérieures}

La non-reconnaissance des acquis et des expériences antérieures est l'élément principal évoqué par plusieurs participantes qui souhaitaient intégrer le marché de l'emploi. En effet, quinze femmes sur vingt disent avoir été confrontées à cet obstacle qui les a amenées à reconfigurer leur nouvelle vie au Canada. L'expérience de la participante A4 illustre cette difficulté. Son expérience ressemble à celle recueillie par Arsenault dans ses travaux :

Plusieurs des femmes interviewées possèdent une formation universitaire ou professionnelle acquise au Congo, mais aussi parfois en Europe. Malgré ce fait, elles se sont toutes heurtées à une fin de non-recevoir au moment de vouloir intégrer le marché du travail dans leur domaine respectif au Québec. (Arsenault, 2003, p. 132)

Elle travaille actuellement en tant que réceptionniste, alors que, dans son pays d'origine, elle avait terminé des études en comptabilité et détenait plusieurs années d'expérience en administration des affaires. Sa famille était détentrice d'une compagnie dont elle était gestionnaire, en s'occupant entre autres de l'embauche d'employés, de leurs rémunérations salariales, etc. Or, l'expérience de cette participante est un exemple qui montre que le fait de venir d'ailleurs, avec des expériences de travail et des compétences professionnelles, ne garantit rien pour elle en matière d'emploi décent, tout comme pour des milliers d'immigrantes, qui veulent intégrer le marché de l'emploi au Canada.

«Pour moi, il n'y avait pas de compagnie qui pouvait m’embaucher, malgré les compétences et les expériences de travail que j'avais dans mon pays. Je gérais mes propres affaires au Congo. J'avais même des employés. Je m’occupais de leurs payes, leurs vacances, etc. Ici, la porte m'était totalement fermée. C'est comme si je n'avais jamais travaillé. C'était à mon tour de me vendre, ce dont je ne connaissais pas. Moi qui offrais du boulot aux employés, c'était à mon tour d'aller chercher le travail. Je ne savais pas comment on demande un emploi au Canada ni par où commencer. » (Participante A4)

"Il est... difficile pour ces femmes de retrouver un emploi similaire à celui quelles occupaient avant leur migration."
Il est donc difficile pour ces femmes de retrouver un emploi similaire à celui qu' elles occupaient avant leur migration. 


\section{Manque de références et d'expérience canadienne}

Le manque de références et d'expérience canadienne constitue un autre défi qui laisse perplexes plusieurs femmes qui détenaient plusieurs années de travail dans leur pays d'origine. Elles sont confrontées à la question de l'expérience de travail au Canada. Par exemple, la participante A2 indique qu'elle avait plusieurs années d'expérience en enseignement dans son pays d'origine et qu'elle a éprouvé des difficultés énormes à intégrer directement le marché du travail canadien. N'ayant pas trouvé de travail dans le domaine de l'éducation, elle travaille actuellement en tant que commis au service à la clientèle. Pour obtenir ce travail, elle a dû retourner aux études. Les expériences antérieures de cette femme, selon elle, n'étaient pas reconnues ni valorisées par les employeurs. De plus, les employeurs lui ont demandé tour à tour de présenter de l'expérience canadienne, ce qu'elle n'a pas encore eu sur le marché du travail canadien.

\section{Conciliation emploi-famille et horaire de travail incompatible}

La conciliation entre la vie familiale et la vie professionnelle est un processus difficile pour les familles en général et pour les femmes en particulier. Dans son analyse intersectionnelle, Guilleux emprunte à Crenshaw la métaphore de l'autoroute, où s'entremêlent plusieurs facteurs, dont le genre.

Les autoroutes représentent les axes majeurs de la discrimination relative à la capacité physique, à la capacité mentale, à la classe, au genre, à la race, à la sexualité, à l'âge et autres sites de discrimination. Le trafic, ce sont les dynamiques entre les différentes discriminations, et le point d'entrecroisement des autoroutes, c'est l'endroit où les différentes discriminations intersectionnelles rencontrent le sujet. (Guilleux, 2015, s.p.)

"...le fait d'être noires, d'être femmes et d'être mères a concomitamment une incidence sur leur capacité de trouver un travail et de rester... sur le marché de l'emploi." "
Pour les femmes interrogées, le fait d'être noires, d'être femmes et d'être mères a concomitamment une incidence sur leur capacité de trouver un travail et de rester durablement sur le marché de l'emploi.

Trouver un emploi n'est pas chose facile lorsqu'on a des enfants et qu'on n'a pas de soutien pour le gardiennage. La situation de la participante A6 illustre cette difficulté à laquelle plusieurs participantes congolaises sont confrontées :

"Quand on m'a proposé l'emploi, on ne m'avait pas dit que je devais travailler les fins de semaine. Je trouvais que l'horaire n'était pas à mon goût. 
J'étais disponible dans la semaine, mais on me donnait congé comme lundi, mardi, pour enfin me demander de travailler la fin de semaine [...] Il faut se débrouiller, mais c'est toujours difficile sans aide. Alors, tu fais un choix entre ta famille et ton travail. J'ai choisi de rester à la maison pour élever mes enfants. » (Participante A6)

Les femmes immigrantes congolaises ne semblent pas être les seules à faire face à cet obstacle qui constitue un frein pour plusieurs femmes qui désirent intégrer le marché de l'emploi. Si les femmes canadiennes rencontrent ce même défi à concilier famille et emploi, pour les femmes immigrantes, ce défi est lourd d'autant qu'elles n'ont pas de proches ni de parents sur lesquels elles peuvent compter pour les épauler dans le gardiennage d'enfants. Contrairement aux Canadiennes de naissance, qui souvent ont de la famille proche et qui peuvent bénéficier de soutiens divers, les femmes congolaises ne doivent compter que sur elles-mêmes.

\section{Barrières bureaucratiques et manque de courtoisie envers les personnes noires}

D'après certaines femmes congolaises, on ne doit pas toujours espérer avoir de bons services lorsqu'on se présente, par exemple, devant les secrétaires pour faire une demande d'emploi à cause de leurs préjugés envers les personnes noires. Certaines participantes mentionnent que, souvent, ces dernières ne sont pas toujours courtoises et ne donnent pas le même service aux personnes noires et aux personnes blanches. Selon elles, certaines sont racistes et intolérantes envers les personnes noires. Pour ce qui est de la réception d'un curriculum vitae, elles peuvent décider d'en disposer comme bon leur semble. La participante A7 fait partie des participantes qui ont été découragées par les services offerts par les secrétaires :

"J'ai trouvé très difficile le manque de courtoisie envers nous, les Noirs, dans les services qu'on donne aux Blancs, comparativement à nous, les Noirs [...] Des fois, ça se passe dans des bureaux, la secrétaire parle avec quelqu'un, elle accueille tout le monde avec enthousiasme, mais quand, toi, ton tour arrive, elle change complètement de face; son sourire disparait, sa courtoisie disparaît, etc. » (Participante A7)

La participante A7 n'est pas la seule à déplorer les services inégaux que les personnes noires obtiennent de la part de secrétaires. En effet, la participante A10, qui avait envoyé son curriculum vitae à la demande d'un employeur, s'est rendu compte, à sa grande surprise, que la secrétaire n'avait jamais fait acheminer son curriculum vitae. 
" " J'ai trouvé ça très difficile quand une secrétaire n'avait pas acheminé mon $C V$ au comité de sélection. Quand jai rappelé, son patron était étonné qu'elle ne lui ait pas soumis mon CV..." "

" J'ai trouvé ça très difficile quand une secrétaire n’avait pas acheminé mon CV au comité de sélection. Quand j’ai rappelé, son patron était étonné qu'elle ne lui ait pas soumis mon CV, alors que c'était lui qui m'avait dit de venir le porter à sa secrétaire. Notre couleur de peau nous cause beaucoup d'obstacles, mais on ne peut rien y faire. Il faut vivre avec. » (Participante A10)

Visiblement, il semble bien que les services que reçoivent les citoyennes et citoyens sont inhérents à la couleur de leur peau, selon le ressenti et le vécu des personnes concernées. Même si cela pourrait alimenter les débats, il n'en demeure pas moins que c'est ce que ressentent ces femmes congolaises immigrées au Canada à travers leur parcours de chercheuses d'emploi. En effet, au Canada, les femmes noires, dont les femmes congolaises, sont ainsi discriminées sur le marché de l'emploi à cause de leur groupe d'appartenance, leur genre et leur couleur de peau :

La question de l'intégration des femmes immigrantes noires au marché du travail au Québec, soulève une triple problématique de discrimination : à l'égard des femmes, à l'égard des minorités visibles, particulièrement à l'égard des gens de souche africaine, et enfin à l'égard des immigrantes et immigrants. (Komoé, 2005, p. 41)

\section{Obstacles rencontrés en cours d'emploi}

Les femmes congolaises disent avoir rencontré différents obstacles à intégrer le marché de l'emploi, confirmant ainsi les données de certains rapports, comme celui de l'Action travail des femmes (ATF) du Québec, qui dit :

Dans toutes les sphères d'activité, les femmes doivent surmonter des obstacles différents de ceux rencontrés par les hommes. Pour certaines femmes, dont les immigrantes, ces obstacles sont liés à diverses formes de discrimination, fondées sur le sexe, la race ou la classe sociale. Officiellement, les femmes jouissent de ces droits au même titre que les hommes, mais l'inégalité entre les sexes persiste, tant dans la politique gouvernementale que sur les marchés du travail. (ATF, 2009, p. 38) 


\section{Difficultés reliées aux discriminations, au racisme et aux préjugés sur le travail des personnes noires}

Au Canada, certaines personnes ont tendance à nier l'existence des discriminations et du racisme. Or, certains actes discriminatoires semblent beaucoup déstabiliser de femmes noires sur le marché de l'emploi. Pourtant, la participante A2 faisait partie de celles qui niaient la présence de ces types d'actes au Canada, jusqu'à ce qu' elle-même en soit victime.

"C'est à partir de mon premier travail que j'ai connu c'était quoi la discrimination au travail et comment ça fait mal. Pourtant, je refusais de croire à la présence des discriminations au Canada [...] Vous vous rendez compte que mes collègues mavaient accusée de vol du tiroir-caisse? Heureusement pour moi, il y avait une caméra pour tout filmer. Ce qui m’avait sauvé la vie. Mes collègues étaient de vraies racistes. » (Participante A2)

La participante A10, qui a migré au Canada alors qu'elle était très jeune, dit avoir souffert des stéréotypes et préjugés sur son travail, alors qu'elle occupait un emploi dans une manufacture où elle se faisait souvent dire que les personnes noires ne travaillent pas vite.

«Les préjugés et les stéréotypes envers les Noirs, j’en ai connu. Par exemple, quand tu entends quelqu' un te crier pour te dire que tu ne travailles pas assez vite. Il va dire : "Les Noirs sont toujours comme ça. Ils ne travaillent pas vite, ils sont lents, ne travaillent pas vite." [...] Pour écarter les Noirs sur le marché de l'emploi, les gens sont prêts à tout inventer. " (Participante A10)

Les femmes congolaises sont également victimes d'injures, de blagues, de moqueries et de commentaires désobligeants sur leurs origines. L'expérience de la participante A10 illustre bien ce défi auquel plusieurs femmes immigrantes sont confrontées.

"Oui, j'ai été victime d'injures et des blagues liées à mes origines et la couleur de ma peau,... Ça peut arriver que quelqu'un te dise de retourner chez toi, il y a beaucoup de Noirs ici [...] Qu'estce que tu es venue faire ici? " "
"Oui, j'ai été victime d'injures et des blagues liées à mes origines et la couleur de ma peau, mais pas avec mon employeur. C'était surtout avec le membre de l'équipe. Ça peut arriver que quelqu'un te dise de retourner chez toi, il y a beaucoup de Noirs ici [...] Qu'est-ce

que tu es venue faire ici? » (Participante A10) 
Il est à noter que ces femmes, sans distinction de leur lieu de naissance, se plaignent d'être victimes de ces phénomènes, qu'elles soient nées au Canada ou qu'elles aient immigré. L'expérience de la participante A1 montre comment les conséquences de ces phénomènes ne font pas de distinction entre les femmes congolaises au Canada. Elles sont ainsi racisées.

«Le marché du travail ne distingue pas une femme noire qui est née ici et celle qui est venue d'un autre pays [...] Quand je cherchais le travail, la première chose que l'employeur remarquait dès que je me présentais, c'est que j'étais une femme noire. Peu importe le temps que j'ai effectué au Canada, pour eux, j'étais noire. Un point c'est tout. » (Participante A1)

Être né au Canada ou au Congo ne fait aucune différence aux yeux des personnes qui discriminent. Le point de vue de la participante A10 associe, entre autres, les discriminations contre les femmes noires à la conjoncture économique. En effet, la conjoncture économique a une incidence sur l'acceptation des personnes noires sur le marché de l'emploi.

" Je crois aussi que c’est étant donné la crise économique que nous sommes discriminées actuellement. Quand il y a crise économique, qu'il y a crise d'emploi, nous, les immigrants, nous sommes plus victimes. Les gens pensent que nous volons leurs emplois, et on a de la misère à nous offrir de l'emploi. » (Participante A10)

Même certaines Congolaises qui n’ont jamais été victimes d'actes racistes dans la vie courante finissent par vivre des actes racistes sur le marché de l'emploi. En d'autres termes, le marché de l'emploi leur a fait changer de perception par rapport au racisme au quotidien. De toute évidence, les préjugés raciaux se manifestent fréquemment autour des stéréotypes à l'endroit des femmes immigrées au Canada tendant à les essentialiser, que ce soit à propos de leurs aptitudes au travail ou par rapport au fait qu'elles sont des Canadiennes venues d'ailleurs.

\section{Compétences linguistiques}

Les difficultés reliées à la maîtrise de la langue anglaise sont un frein pour les femmes congolaises sur le marché de l'emploi. Plusieurs d'entre elles ont préféré s'installer au Québec, étant donné sa particularité pour la langue dominante française. Cependant, il faut noter que, bien que la province de Québec soit la province au Canada où la majorité des personnes parlent le français, dans certaines régions de cette province, l'usage de la 
langue anglaise semble être prédominant et utilisé dans plusieurs domaines d'emploi. C'est un détail qu'ignorent certaines femmes congolaises qui se trouvent confrontées à l'obstacle de la langue anglaise, en particulier quand elles désirent postuler pour des emplois dans les secteurs où la clientèle ne parle pas français. L'expérience de la participante A6 illustre bien cette difficulté :

" "J'ai trouvé un travail, mais je ne pouvais pas parler en anglais. Je me suis sentie comme étant la proie de curiosité. Tout le monde qui arrivait là, on te regarde, on te regarde; ils se demandaient comment toi, une femme noire, tu es là... "

"J'ai trouvé un travail, mais je ne pouvais pas parler en anglais. Je me suis sentie comme étant la proie de curiosité. Tout le monde qui arrivait là, on te regarde, on te regarde; ils se demandaient comment toi, une femme noire, tu es là [...] Selon moi, c'était une discrimination raciale que les gens se questionnent sur comment, moi, j'étais arrivée là. C'est de la discrimination raciale [...] Les gens voulaient m'intimider pour voir comment je vais faire quand ils maborderont en anglais. » (Participante A6)

La barrière linguistique, en ce qui concerne l'anglais, est un facteur de discrimination de ces femmes. Ces barrières ont une grande incidence sur la vie de ces femmes, surtout si elles les intègrent elles-mêmes dans leur comportement et dans leur pensée individuelle et collective (Carpentier, 2011, p. 20).

\section{Conciliation famille-travail et manque de soutien pour les femmes seules}

Parmi les défis rencontrés par les femmes congolaises avant d'entrer sur le marché de l'emploi, on peut faire état de la conciliation familiale. Cette dernière semble se répercuter dans le parcours professionnel de plusieurs femmes immigrantes. C'est le cas de la participante A5, qui a migré seule avec ses enfants, laissant derrière elle son mari, dont les papiers d'immigration n'étaient pas encore prêts.

"J'ai trouvé très difficile de travailler avec des enfants, en charge toute seule [...] Je n'avais personne pour m'aider à garder les enfants. Alors j'ai trouvé ça très difficile de quitter en pleine nuit pour aller travailler et laisser mes enfants seuls [...] J'ai donc décidé de démissionner. " (Participante A5)

Tout comme la majorité des familles monoparentales canadiennes, les femmes congolaises immigrées en situation de monoparentalité au Canada ont vécu ou vivent les mêmes vicissitudes quand il s'agit de faire garder leurs enfants afin d'aller travailler. 


\section{Isolement de la part de collègues de travail}

"...l'isolement semble être une astuce couramment utilisée par des collègues qui ne veulent pas travailler avec des femmes immigrantes,..."

Selon les participantes, l'isolement semble être une astuce couramment utilisée par des collègues qui ne veulent pas travailler avec des femmes immigrantes, notamment les femmes noires. Elles disent souffrir de ce problème qui les place dans des situations où elles sont obligées d'exécuter seules une tâche qui, pourtant, exige l'aide d'une coéquipière ou d'un coéquipier. La participante A14 illustre bien cette difficulté :

"Des fois, j’ai travaillé avec les équipes des autres étages, mais ce n’était pas facile. Ce n'était pas un bon travail d'équipe [...] Mais avec elles, je suis convaincue que c'était vraiment de la discrimination, parce que s'il y a des Noires, elles ne peuvent pas vraiment les aider. Elles vont juste s'aider entre elles, mais, toi, elles te laissent toute seule [...] C'est seulement avec les Noirs qu'elles faisaient ça. » (Participante A14)

Les femmes immigrées congolaises vivent ainsi une forme d'isolement, que ce soit à travers des stratégies conscientes ou inconscientes de la part de leurs collègues qui ne sont souvent pas solidaires d'elles. L'esprit d'équipe se manifeste visiblement à géométrie variable quand il s'agit des Noires, selon les propos de la participante A14.

\section{Intimidation, harcèlement et menaces de congédiement}

"... les fermmes congolaises disent avoir eu à composer avec diverses formes d'abus de pouvoir de la part de leurs collègues ou de leurs supérieurs..."
En plus de l'isolement au travail, les femmes congolaises disent avoir eu à composer avec diverses formes d'abus de pouvoir de la part de leurs collègues ou de leurs supérieurs, qui les intimidaient et les menaçaient de qui les intimidaient et les mé
qunte A18 montre cette difficulté :

"Beaucoup de fois, quand l'erreur est faite par une infirmière noire, les politiques et procédures de l'établissement sont appliquées à la lettre. Mais quand c'est une infirmière blanche, même si l'erreur est plus grave que celle de l'infirmière noire, la superviseure peut même déchirer le rapport de déclaration de l'incident pour protéger l'infirmière blanche. Nous vivons vraiment de la discrimination. » (Participante A18) 
Les participantes qui travaillent dans le domaine de la santé, par exemple en tant qu'infirmières, disent marcher sur des œufs, car leurs emplois sont précaires. Elles peuvent perdre leur emploi n'importe quand, et ce, à cause de la faute d'une autre personne ou des intimidations des autres employés. Plusieurs d'entre elles sont obligées de redoubler de vigilance, car la moindre erreur causée par une distraction ou une manœuvre d'un autre employé peut leur coûter leur travail. La participante A8 a connu une expérience un peu corsée avec sa collègue, qui n'était pas d'accord avec elle sur l'expression médicale qu'elle avait employée.

" "Comme je dis, les collègues blanches, elles ont tendance à se croire supérieures à nous et à vouloir nous contrôler... Elles croient que nous sommes ignorantes et que nous ne pouvons rien leur apprendre. "»
"Comme je dis, les collègues blanches, elles ont tendance à se croire supérieures à nous et à vouloir nous contrôler. Elles ne veulent pas du tout qu'on leur dise qu'on connaît quelque chose, que ce soit sur les fonctionnements de machines ou quelques expressions dans le domaine de

travail. Elles croient que nous sommes ignorantes et que nous ne pouvons rien leur apprendre. » (Participante A8)

Certains employeurs vont jusqu'à abuser de la vulnérabilité d'employées qui sont jeunes et qu'ils intimident avec des menaces de congédiement, si elles ne répondent pas à leurs exigences. Le cas de la participante A13 est particulier. Elle s'est fait embaucher par un employeur qui espérait avoir des avantages envers ses jeunes employées, dont celui de sortir avec elles. Elle s'est trouvée être victime d'intimidation et de harcèlement de la part de son employeur qui s'intéressait aux jeunes filles qu'il embauchait.

"J'étais jeune, mais j'ai constaté que mon patron n'était pas respectueux. Voir un homme de 40 ans et plus, ou de l'âge de mon père, en train de me draguer et de me mettre mal à l'aise, je trouvais ça pas bon pour moi [...] J'ai trouvé son comportement comme du harcèlement. » (Participante A13)

Les difficultés rencontrées par des femmes immigrées d'origine congolaise vivant au Canada sont multiples sur le marché du travail. Lorsqu'elles ont la chance de trouver un emploi, elles doivent se battre pour le garder, car la moindre faute de leur part est amplifiée. Malgré tous les efforts qu'elles déploient, leurs collègues blanches se croient toujours plus compétentes qu'elles, du seul fait d'être blanches. C'est ce que dit en substance la participante A8 dans les termes qui suivent : 
"J'avoue que mes relations avec mes collègues de travail, parfois ça chauffe entre nous [...] Je ne sais jamais quoi attendre de mes collègues. Elles ont toujours des accusations à faire à propos de moi. Je suis toujours convoquée par mes supérieurs pour m'expliquer, mais, comme ils savent que je travaille bien et que je respecte les autres, leurs accusations, c'est de la peine perdue. » (Participante A8)

"Souvent, les patrons veulent aussi, quant à eux, profiter de leur vulnérabilité. De peur de perdre leurs emplois, les femmes congolaises se doivent d'être très vigilantes..."

Souvent, les patrons veulent aussi, quant à eux, profiter de leur vulnérabilité. De peur de perdre leurs emplois, les femmes congolaises se doivent d'être très vigilantes dans la mesure où la moindre erreur de leur part peut entraîner la perte de leur travail. Pour cette raison, elles sont toujours obligées de contrôler leur tempérament pour ne pas s'engager dans des conflits et gageures qui pourraient nuire à la réputation de travailleuses et travailleurs noirs. Bref, elles ne veulent tout simplement pas perdre leurs emplois. L'expérience de la participante A8 montre cette difficulté qui est arrivée à plusieurs participantes. Pour ne pas compromettre leurs emplois et la chance d'autres personnes noires de trouver $\mathrm{du}$ travail, ces femmes doivent tout faire pour éviter disputes et querelles.

"Je sais que leur plan, c'est de me nuire, mais je ne veux pas leur donner cette chance-là. Aussi, je ne veux pas fermer la chance aux autres Noirs de trouver du travail là. Alors, je reste calme et je m'éloigne des querelles et des chicanes, mais c'est très difficile. » (Participante A8)

Les interactions qu'ont ces femmes en milieu de travail sont souvent exécrables. Cependant, de peur de nuire aux autres femmes, elles font preuve de mutisme et de résilience. C'est la preuve qu'elles sont solidaires par rapport aux autres migrantes en quête d'emplois éventuels.

\section{Difficulté à obtenir une promotion ou la permanence au travail}

Au Canada, certains groupes de femmes éprouvent énormément de difficulté à obtenir une promotion au travail ou à obtenir une permanence, comme le pense la participante A18. Cette réalité semble s'aggraver chez les femmes immigrantes noires qui désirent promouvoir leur carrière. L'expérience de la participante A18 permet d'illustrer cette difficulté. Elle travaille dans le domaine hospitalier, où elle occupe actuellement un poste en administration. 
"L'administration est un gang de gens qui font ce qu'ils veulent et comme ils veulent. Tu peux chercher à t'épanouir, mais ils sont prêts (et capables) à te maintenir à la place où tu es [...] C'est très difficile d'avancer dans ta carrière quand tu es noire [...] Pour eux, les Noirs n'ont pas le droit d'évoluer. L'évolution de femmes noires ne compte pas pour eux. » (Participante A18)

L'expérience de la participante A9 montre comment un directeur ou un superviseur peut constituer un blocage pour une femme qui veut avancer dans sa carrière. Cette participante travaille actuellement dans le domaine de l'éducation. Elle dit avoir manqué une opportunité d'obtenir une permanence à l'école où elle travaillait, à la suite des pièges tendus contre elle par ses collègues et son directeur. Ce dernier n'avait jamais voulu qu' elle soit permanente dans son école.

«Mon directeur n'avait jamais voulu que j'occupe le poste laissé vacant par l'enseignante que je remplaçais. Il souhaiterait n'importe qui à ce poste, sauf moi. La Commission scolaire exigeait qu'il présente mon évaluation, mais il ne l'a jamais fait. Jusqu'à ce que mon dossier soit fermé à la Commission [...] La Commission scolaire ne pouvait pas considérer ma candidature sans qu' elle ait obtenu mon évaluation. Ma demande a été ainsi rejetée. » (Participante A9)

Les difficultés d'intégration rencontrées par les femmes immigrées d'origine congolaise constituent de vrais obstacles pour leur promotion professionnelle. Que ce soit au niveau des administrations ou auprès des superviseurs, la promotion de la carrière des femmes congolaises n'est pas la priorité, selon la participante A9. La discrimination à l'égard de ces femmes est ouverte ou insidieuse. C'est ce que Chicha (2009) a d'ailleurs confirmé en indiquant comment la discrimination en milieu de travail peut se présenter de façon très ouverte ou de façon subtile, notamment sous la forme de pratiques de gestion des ressources humaines qui ont un effet négatif disproportionné en fonction du sexe, etc. Et pourtant, ces femmes congolaises adoptent des stratégies de résilience.

\section{" II faut se battre "}

Les expériences des femmes congolaises et certains écrits, dont celui de l'Action travail des femmes du Québec, confirment que, dans toutes les sphères d'activité, les femmes doivent surmonter des obstacles différents de ceux rencontrés par les hommes. Pour les immigrantes, ces obstacles sont inhérents aux diverses formes de discrimination fondées sur le sexe, la race ou la classe sociale. Même si, officiellement, les femmes ont les mêmes droits que les hommes, l'inégalité entre les sexes persiste, tant dans la politique 
gouvernementale que sur le marché du travail (ATF, 2009, p. 38). Par conséquent, quand on est une femme immigrante, noire et d'origine africaine, pour parvenir à intégrer le marché de l'emploi au Canada, cela exige d'être résiliente, courageuse et patiente pour faire face aux épreuves et obstacles que représente la recherche d'un travail au Canada. Comme en témoignent quinze des vingt participantes, " il faut se battre ». Mais que veut dire « il faut se battre », pour ces femmes congolaises?

\section{Se battre pour trouver du travail et le conserver}

"... les femmes congolaises parlent souvent de "se battre ", en particulier lorsquelles évoquent leurs expériences de recherche de travail."
Durant les entretiens, les femmes congolaises parlent souvent de "se battre ", en particulier lorsqu'elles évoquent leurs expériences de recherche de travail. Selon elles, quoique cette phrase ne soit qu'une expression et bien qu'il n'y ait pas de guerre au Canada, elles ont l'impression qu'elles sont constamment en train de se battre, notamment pour trouver du travail et surtout pour le conserver. L'expérience de la participante A16 explique ce que signifie le terme "se battre ", car, selon elle, il est utilisé afin de montrer qu'il ne faut pas rester inerte, qu'il faut changer sa situation.

"C'est un terme qui est employé, sinon te battre comment? Tu ne vas pas aller dans la rue pour te battre avec quelqu'un. Moi, je peux dire qu'on se bat pour se faire une place au soleil. Ça veut dire quoi en vrai sens, en vrai terme, il faut savoir te fixer un objectif. Je suis venue au Canada. Dans mon pays, il y a la guerre, le pays est en désordre [...] C’est une expression, en général, on dit ça, mais je t'assure que c'est un combat que nous menons au Canada. Il faut se battre pour tout avoir. » (Participante A16)

Les femmes congolaises immigrées au Canada se construisent alors un mental de combattante devant l'adversité. Pour elles, cela constitue une sorte de bouée de sauvetage en plus d'être un facteur de motivation. Par ailleurs, pourquoi et contre qui « se battre »? Les femmes congolaises ont plusieurs raisons qui les poussent à se battre. La précarité des emplois et la compétition entre les candidates ou candidats aux postes disponibles exigent qu'elles puissent se démarquer pour être recrutées puisque, selon l'Organisation internationale du travail (OIT), les nets progrès accomplis par les femmes dans l'éducation ne se sont pas traduits par une amélioration comparable de leur situation professionnelle (OIT, 2016). Ce qui est vrai pour les femmes, en général, l'est encore plus pour les femmes noires immigrées (ATF, 2009). L'expérience de la participante A6 montre comment la 
compétition entre collègues et les pièges que ces derniers tendent aux femmes immigrantes font en sorte qu'elles n'ont pas d'autre choix que de se battre.

"Oui, il faut se battre, parce que si tu ne te bats pas, mais tu ne peux pas trouver un travail facile ici dans ce pays. Il faut se battre, parce qu'il ne faut pas se laisser faire. Parce que si tu arrives dans un milieu où il y a quatre ou cinq Québécois ou Canadiens, tu vas voir, souvent, elles se font des groupes contre toi et ne te parlent pas. Tu es isolée, seule dans ton coin. Seule pour travailler et parfois seule pour manger. Personne ne te parle. Si tu n'as pas le cœur solide, tu quittes et tu ne reviens plus le lendemain [...] Les femmes congolaises ne devraient pas perdre de vue qu' il faut se battre. " (Participante A 6)

"Si, pour certaines Congolaises, il leur faut lutter contre les intolérances de leurs collègues qui les isolent au travail, pour d'autres, il faut le faire pour soimême, pour sa famille et pour sa communauté qui est victime des préjugés. »
$\mathrm{Si}$, pour certaines Congolaises, il leur faut lutter contre les intolérances de leurs collègues qui les isolent au travail, pour d'autres, il faut le faire pour soi-même, pour sa famille et pour sa communauté qui est victime des préjugés. L'expérience de la participante A17 explique bien cette observation :

" Je me bats pour moi-même; je me bats aussi pour mes enfants, pour ma communauté noire, pour qu'ils puissent voir qu'il n'y a pas d'obstacles qu'on ne peut pas vaincre. Quand on cherche quelque chose, on doit y tenir et se donner les moyens pour y arriver. Il y a beaucoup de préjugés sur nous, et les gens pensent que nous, les Noirs, on ne peut pas avoir le meilleur [...]" (Participante A17)

Donc, les femmes congolaises ont l'impression de ne pas être traitées comme leurs collègues blanches, au travail. C'est ainsi que s'est sentie la participante A5 qui, pour plaire à son chef d'équipe, qui lui assignait de lourdes tâches que son directeur lui interdisait d'exécuter, a dû travailler fort au point d'avoir un accident de travail. Elle est actuellement invalide et doit suivre des séances de physiothérapie pour l'aider à recouvrer sa santé.

"Mon travail me faisait penser au temps de l'esclavage, où le maître était derrière son esclave et le fouettait pour qu'il accélère le travail. La grande différence avec mon travail, c'est que mon chef d'équipe n'avait pas de fouet pour me taper, mais c'était comme si j'étais son esclave. » (Participante A5) 
Contrairement à cette participante qui se comparait à l'esclave de son superviseur qui exerçait de la pression sur elle, la participante A7 avait l'impression d'être considérée comme une machine à tout faire.

"Comme nous, les femmes noires, on est comme des esclaves qu'on est des machines à tout faire, on est comme des choses capables de tout faire. Les gens pensent qu'on est capables de faire toutes les tâches comme des machines. Alors, on fait appel à nous [...]» (Participante A7)

Le fait de se faire constamment appeler " négresse " au travail et dans leur société d'accueil est un comportement qui anéantit plusieurs d'entre elles en quête de travail. Six participantes sur vingt disent avoir entendu les gens les appeler " négresse ", alors qu'ils connaissaient bien leur nom. La participante A4 dit maintenant comprendre les effets de ce mot et ses conséquences sur une femme noire.

"C'était une journée normale. Je ne sais pas si je me suis réveillée ce jour-là d'un mauvais côté, mais la journée s'est mal déroulée. J'arrive à la clinique, j’y trouve une patiente qui était de mauvaise humeur, je pense, elle s'est présentée devant mon poste et m'a traitée de tous les mots dont "la négresse". "

Dans le milieu de la santé, les patients lucides ne sont pas les seuls à dénigrer les femmes noires en les traitant de "négresses». Ceux qui souffrent de démence ou de troubles mentaux emploient souvent ce mot pour perturber ces femmes dont ils supportent mal la présence. La participante A14 dit avoir entendu résonner ce mot dans ses oreilles à plusieurs reprises, au point où, quand elle se faisait traiter de "négresse ", la seule réponse qu'elle donnait à son adversaire c'est : " Je suis fière d'être "négresse". "

"Mon expérience de travail était bien, mais de dire avec les patientes, ce n'était pas facile. Soit tu entres dans la chambre, on te chasse, tu entres dans la chambre, la patiente dit: "Je ne veux pas te voir, parce que tu es noire. Négresse, je ne veux pas que tu me touches, tu es sale." " "
"Mon expérience de travail était bien, mais de dire avec les patientes, ce n'était pas facile. Soit tu entres dans la chambre, on te chasse, tu entres dans la chambre, la patiente dit: "Je ne veux pas te voir, parce que tu es noire. Négresse, je ne veux pas que tu me touches, tu es sale." " (Participante A14)

Devant l'impuissance de la plupart de ces femmes congolaises d'être valorisées et respectées par les collègues, les employeurs et les personnes à qui elles donnent des soins 
en tant qu'infirmières ou préposées aux bénéficiaires, elles n'avaient d'autre choix que de développer différentes stratégies pour survivre au travail et garder leur emploi. L'une des stratégies utilisées pour survivre au travail ou garder leur emploi est d'avoir recours aux parents qui vivent toujours dans le pays d'origine dans leur quête de réconfort. Le témoignage de la participante A5 montre ce défi de recourir à la famille. En effet, elle a eu recours à sa mère afin qu'elle puisse l'encourager à traverser les expériences difficiles au travail.

" Je suis déjà arrivée que je dois appeler ma mère pour lui parler de ce que je vis au travail, pour avoir son réconfort. J'arrive même à partager ma peine avec ma mère qui n'est pas ici et qui m'encourage de continuer, car on n'est pas chez nous. Elle me dit "courage". C'est ça la vie. Comme tu as des besoins, tu vas tout supporter, et puis on n'est pas chez nous. " (Participante A5)

Elles sont toutes convaincues que la persévérance est importante comme moyen de résilience afin de traverser des moments difficiles au travail. La participante A5 a opté pour la persévérance, alors qu'elle travaillait avec un superviseur qui la méprisait au travail. Ce dernier connaissait son nom, mais il ne l'appelait jamais par son nom.

"Mon superviseur était vraiment irrespectueux, mais je n'avais pas de choix de travailler dans de telles conditions. Pour rester, j'étais persévérante; j'ai trouvé très difficile de travailler avec quelqu'un qui me traitait de la sorte. J'étais sans importance pour lui. J'avais même perdu mon nom. Il me disait "hé, hé" et j’acceptais. Des fois, je pleurais et je faisais semblant que tout va bien. » (Participante A5)

Plusieurs femmes congolaises sont obligées d'endurer des conditions très difficiles au travail, où elles doivent persévérer devant les comportements de leurs collègues et superviseurs qui sont irrespectueux et qui leur enlèvent leur dignité.

"Elles se battent d'abord pour elles-mêmes et ensuite pour leur progéniture, pour leur groupe ethnique ou "racial ", voire pour leur pays d'origine. Elles se battent surtout pour la dignité humaine..."
En définitive, plusieurs mobiles soustendent le combat des femmes congolaises. Elles se battent d'abord pour elles-mêmes et ensuite pour leur progéniture, pour leur groupe ethnique ou " racial ", voire pour leur pays d'origine. Elles se battent surtout
assé très peu glorieux de l'esclavage connu pour la dignité humaine en se rappelant le passé très peu glorieux de l'esclavage connu par leurs ancêtres. 


\section{Stratégies mobilisées pour se battre}

Les femmes noires, comparativement aux femmes blanches, souffrent de plus grands obstacles et défis qui sont imposés par les sociétés dominantes et patriarcales, mais qui n'affectent pas les femmes blanches au même point. Les femmes blanches ne connaissent pas les mêmes contraintes que les femmes racisées, comme le soulignent Corbeil et Marchand :

Même si les femmes blanches et les femmes racisées subissent les contrecoups $\mathrm{du}$ patriarcat, les premières ont des privilèges que les secondes n'ont pas en raison du racisme et de ses effets subséquents : statut socioéconomique précaire, ghettoïsation, isolement, dévaluation professionnelle, etc. En d'autres termes, les femmes blanches, principalement celles qui sont issues des classes moyenne et supérieure, peuvent bénéficier de ressources auxquelles les femmes racisées n'ont pas forcément accès. (Corbeil et Marchand, 2007, p. 5-6)

En ce qui concerne les femmes congolaises précarisées, racisées, isolées et dévaluées professionnellement, il n'y a pas beaucoup de portes de sortie. Plusieurs des participantes étaient partagées entre les stratégies individuelles et les stratégies collectives, mais, selon elles, les unes n'empêchent pas les autres. D'autres femmes se sentent tout simplement abandonnées face aux exigences du marché de l'emploi. Ainsi, elles se conforment selon les circonstances. Et pourtant, elles sont quand même convaincues que c'est grâce à des manifestations et stratégies collectives qu' elles parviendront à vaincre les discriminations et injustices dont elles sont victimes, comme le soutient la participante A1 :

"C'est en se battant collectivement que nous arriverons à valoriser notre image en tant que femmes noires et africaines. Etre fières d'être Noires et Africaines. $\hat{E}$ tre solidaires et montrer que nous sommes unies et nous nous battons pour la même cause. "
«C'est en se battant collectivement que nous arriverons à valoriser notre image en tant que femmes noires et africaines. Être fières d'être Noires et Africaines. Être solidaires et montrer que nous sommes unies et nous nous battons pour la même cause. Avec nos forces unies, nous allons avoir la victoire pour les générations des femmes. " (Participante A1)

Sentant qu'elles ne peuvent pas gagner ce combat à elles seules, certaines femmes congolaises reconnaissent la nécessité d'une mobilisation collective entraînant une lutte globale et collective. Toutefois, le marché de l'emploi ne semble pas être le seul secteur 
qui demande la mobilisation des stratégies pour mettre fin entre autres aux inégalités et injustices qui affligent certains groupes sociaux, dont les personnes noires. Ceci se reflète de plus en plus dans plusieurs sociétés, tout particulièrement par des mouvements de solidarité en faveur des personnes les plus vulnérables, par exemple le mouvement Black Lives Matter (La vie des Noirs compte), qui s'insurge contre la violence et le racisme des forces de l'ordre envers les personnes noires dans nos sociétés.

\section{Conclusion}

Bilge (2009) propose de considérer l'intersectionnalité comme un grand principe qui pourrait être ajusté et complété en fonction des champs d'étude et des objectifs de recherche, afin d'en faire une mise en application diverse et plurielle. C'est justement dans cette optique que se place cet article en utilisant ce concept dans le champ de la sociologie (des migrations et des inégalités). L'analyse intersectionnelle s'ajuste donc dans son application plurielle et a permis dans cette perspective de traiter des notions comme l'exclusion, les inégalités, la discrimination, le racisme en plus de la question du genre. Au-delà des critiques du concept de l'intersectionnalité par rapport au féminisme, il demeure un outil d'analyse pertinent surtout sur les sujets touchant les inégalités et les discriminations. Les concepts mobilisés sont relatifs aux marqueurs identitaires, en particulier la couleur de la peau, l'origine ethnique et le sexe. Le tout est inséré dans une approche multidimensionnelle. Comme l'a dit Hyppolite dans l'une de ses publications :

Les femmes immigrées, particulièrement celles des minorités visibles, connaissent des difficultés en ce qui a trait à leur insertion professionnelle. Le racisme et le sexisme combinés créent des situations d'inégalité dans le domaine de l'emploi. Beaucoup d'employeurs jugent les femmes immigrées d'après la couleur de leur peau et entretiennent des préjugés sur leurs habitudes de travail, sur leurs aptitudes à occuper certains emplois et sur leurs capacités d'intégration. (Hyppolite, 2012, p. 247)

Les propos d'Hyppolite (2012) se confirment chez les femmes immigrées congolaises. Mais en plus, ces femmes font preuve à la fois de résilience et de combativité face au schisme entre leurs espoirs et la réalité après avoir concrétisé leur projet d'immigration en Ontario et au Québec. Dans le cadre du processus migratoire des femmes congolaises au Canada, les données recueillies montrent suffisamment les perceptions qu'elles ont de leur vécu. D'où la nécessité pour elles de s'approprier le combat à travers l'idée qu'elles expriment : 
"Il faut se battre. » En effet, après les femmes autochtones, les femmes africaines sont les personnes les plus défavorisées de la société canadienne, ce qui a de grandes répercussions sur leur avenir et celui de leurs enfants. Malgré tous leurs efforts, l'intolérance et le racisme envers ces femmes restent palpables, tant sur le marché de l'emploi que dans la vie quotidienne, dont le milieu scolaire. Si les travaux de Pierre (2005, p. 84) ont pu démontrer globalement les facteurs d'exclusion qui font obstacle à l'intégration socioéconomique de certains groupes de femmes immigrées et noires au Québec, notre étude et notre analyse, quant à elles, ont une portée à la fois large et spécifique. Elles ont une portée large, car elles s'étendent géographiquement sur le Québec et l'Ontario (au Canada). Elles ont une portée spécifique dans la mesure où elles se sont intéressées aux femmes immigrantes congolaises.

"Cet article montre à travers les cas étudiés que les obstacles à l'insertion professionnelle des femmes congolaises sont multifactoriels. Il confirme aussi les travaux de Pierre (2005), selon qui les états multiples de discrimination avec lesquels les immigrantes doivent composer sont intrinsèquement liés à leur genre (leur sexe) et à leur couleur de peau et à leur origine ethnique."
Cet article montre à travers les cas étudiés que les obstacles à l'insertion professionnelle des femmes congolaises sont multifactoriels. Il confirme aussi les travaux de Pierre (2005), selon qui les états multiples de discrimination avec lesquels les immigrantes doivent composer sont intrinsèquement liés à leur genre (leur sexe) et à leur couleur de peau et à leur origine ethnique. Les données collectées montrent après analyse que ces femmes immigrées congolaises ne demandent qu'à être considérées comme des personnes qui ont le droit d'aspirer à un rêve, dont celui d'avoir un travail pour mieux se sentir valorisées, se prendre en charge et contribuer à l'essor du Canada.

L'une des particularités de la présente analyse est de s'être appuyée sur l'intersectionnalité pour saisir les inégalités, sans être inscrite dans un militantisme porteur de clivage. Elle révèle aussi que le parcours difficile, voire chaotique, de ces femmes sur le marché de l'emploi devient à l'inverse leur motif de motivation pour se battre pour leur réussite professionnelle ou pour leur dignité. Les divers discours recueillis et analysés montrent en plus que les femmes congolaises, loin de constituer un groupe homogène, ont des parcours de vie spécifiques. Cependant, leur problème demeure un fait social qu'il a fallu considérer à la fois dans sa globalité et dans sa spécificité avec une méthodologie précise. Ainsi, l'intersectionnalité, privilégiée dans les milieux militants comme académiques pour désigner cette complexe articulation des identités-inégalités multiples, a soutenu le cadre référentiel, conceptuel et méthodologique (Bilge, 2009). L'intersectionnalité a alors permis de découvrir que l'expression « il faut se battre » est à la fois une forme de philosophie de 
vie et une barrière de protection, voire une forme d'auto-motivation, pour les femmes immigrées congolaises en Ontario et au Québec et peut-être ailleurs au Canada.

\section{Bibliographie}

ACTION TRAVAIL DES FEMMES (2009). La reconnaissance des diplômes et des compétences: difficultés et impacts chez les femmes immigrantes, Rapport de recherche-action, réf. du 30 mai 2018, http:// www.cdeacf.ca/actualite/2009/11/19/reconnaissance-diplomes-competences-difficultes-impactschez

ANTIOPE, Nathalie (2008). "Elsa Dorlin, dir., Black Feminism. Anthologie du féminisme africainaméricain, 1975-2000", Questions de communication, 15 | 2009, 424-426, réf. du 16 janvier 2012, https://journals.openedition.org/questionsdecommunication/812

ARSENAULT, Stéphanie (2003). "La séparation et réunification familiales de dix femmes réfugiées congolaises ", Service social, Vol. 50, № 1, p. 122-144.

BLACK LIVES MATTER (2016). « Le mouvement Black Lives Matter expliqué en trois minutes », Le Temps du 11 juillet 2016, réf. du 30 mai 2018, Letemps.ch/monde/mouvement-black-livesmatter-explique-trois-minutes

BERTAUX, Daniel (2010). Le récit de vie. L'enquête et ses méthodes, $3^{e}$ édition, Paris, Armand Colin, 129 p.

BERTAUX, Daniel (1980). « L'approche biographique, sa validité méthodologique, ses potentialités », Cahiers internationaux de sociologie, Vol. 27, № 69, p. 197-225.

BALS, Myriam (1999). Les domestiques étrangères au Canada. Esclaves de l'espoir, Paris/Montréal, L'Harmattan, Logiques sociales, $240 \mathrm{p}$.

BILGE, Sirma (2009). "Théorisation féministe de l'intersectionnalité », Diogène, № 225, p. 70-88.

CARPENTIER, Marie (2011). La discrimination systémique à l'égard des travailleuses et travailleurs migrants", Cat. 2.120-7.29, Québec, Commission des droits de la personne et des droits de la jeunesse, réf. du 30 mai 2018, http://www.cdpdj.qc.ca/publications/Avis_travailleurs_immigrants.pdf

CHICHA, Marie-Thérèse (2009). Le mirage de l'égalité : les immigrées hautement qualifiées à Montréal, Montréal, Fondation canadienne des relations raciales, $136 \mathrm{p}$.

COMMISSION ONTARIENNE DES DROITS DE LA PERSONNE (2005). « Politique et directives sur le racisme et la discrimination raciale ", réf. du 30 mai 2018, http://www.ohrc.on.ca/sites/ default/files/attachments/Policy_and_guidelines_on_racism_and_racial_discrimination_fr.pdf

CORBEIL, Christine, et Isabelle MARCHAND (2007). Lintervention féministe intersectionnelle : un nouveau cadre d'analyse et d'intervention pour répondre aux besoins pluriels des femmes marginalisées et violentées, réf. du 30 mai 2018 , https://unites.uqam.ca/arir/pdf/interventionfeminineintersectionnelle_marchand_corbeil.pdf 
CRENSHAW, Kimberlé W. (2005). "Cartographies des marges : intersectionnalité, politique de l'identité et violences contre les femmes de couleur ", Cahiers du Genre, № 39, p. 51-82.

CYBERSOLIDAIRES (2005). Quelques femmes noires, réf. du 24 février 2005, cybersolidaires.typepad. com/ameriques/2005/02/quelques_femmes.html

DAVIS, Angela (1981). Women, Race \& Class, New York. Vantage Books. Random House, Social science, $271 \mathrm{p}$.

DAVIS, Kathy (2015). L'intersectionnalité, un mot à la mode. Ce qui fait le succès d'une théorie féministe, réf. du 15 juin 2015, https://journals.openedition.org/cedref/827

de CURRAIZE, Yves, et Réjane HUGOUNENQ (2004). "Inégalités de salaires entre femmes et hommes et discrimination ", L'Observatoire français des conjonctures économiques (OFCE), № 90, p. 193-224.

DORLIN, Elsa (2005). "De l'usage épistémologique et politique des catégories de "sexe" et de "race" dans les études sur le genre ", Cahiers du Genre, No 39, p. 83-105.

EID, Paul (2012). «Mesurer la discrimination à l'embauche subie par les minorités racisées : résultats d'un "testing” mené dans le Grand Montréal ", Cat. 2.120-1.31, Québec, Commission des droits de la personne et des droits de la jeunesse.

GILMORE, Jason (2008). «Les immigrants sur le marché du travail canadien en 2006 : analyse selon la région ou le pays de naissance ", document analytique, № 71-606-X2008002 au catalogue, Ottawa, Statistique Canada, 43 p.

GUILLEUX, Céline (2015). Les discriminations multiples et croisées. Multiple and crossed discriminations, réf. du 30 octobre 2015, http://calenda.org/338928

HERLA, Roger (2010). Violence conjugale et intersectionnalité, Collectif contre les violences familiales et l'exclusion (CVFE), réf. du 30 octobre 2015, http:/www.cvfe.be/sites/default/files/doc/EP2010-5-RogerHerla-Intersectionalite-Synth.pdf

hooks, bell (1981). Ain't i a woman: black women and feminism, Boston, South End Press.

hooks, bell (1984). Feminist theory: from margin to center, Boston, South End Press.

HYPPOLITE, Ismaëlie (2012). "Contribution au développement socioéconomique de la région d'Edmonton par les femmes africaines noires francophones immigrées entre 2000 et 2006 ", International Journal of Canadian Studies / Revue internationale d'études canadiennes, $\mathrm{N}^{\circ}$ 45-46, p. 239-259.

IMBOUA, Ginette (2012). Discrimination raciale au sein des femmes immigrantes sur le marché du travail : le cas des minorités visibles et des minorités non visibles de Montréal, [mémoire de maîtrise], Université d'Ottawa, $80 \mathrm{p}$.

INSTITUT CANADIEN DE RECHERCHES SUR LES FEMMES (2003). " Les femmes immigrantes et réfugiées ", Feuillet d'information de l'ICREF, № 5, Automne, 12 p. 
KOMOÉ, Maraki (2005). Lintégration des femmes immigrantes au marché du travail au Québec, [mémoire de maîtrise], Université du Québec à Montréal, 162 p.

LEMOINE, Maryse (2010). "Discrimination et traitement préférentiel envers la communauté francophone immigrante : la recherche de logement des immigrants français et congolais à Toronto ", Relier, relayer, relater les francophonies d'Amérique, No 29, printemps 2010, réf. d'août 2011. https://www.erudit.org/fr/revues/fa/2010-n29-fa1813921/1005417ar.pdf

LY, Aoua Bocar (2007). Etre femme et noire africaine au Québec, réf. du 30 mars 2007, www.continent premier.com/?article $=1211$ \&magazine $=36$

MARTUCELLI, Danilo, et François de SINGLY (2009). Les sociologies de l'individu, sociologies contemporaines, Paris, Armand Colin, coll. «128», 127 p.

MIANDA, Gertrude (1998). «Être une immigrante noire africaine francophone à Toronto : vécu et perception des rapports de genre ", Reflets : revue ontaroise d'intervention sociale et communautaire, Vol. 4, No 1, p. 34-52.

NAVES, Marie-Cécile (2016). "L'inégalité du fait de l'apparence ", réf. du 17 février 2016.

NIKUZE, Christine (2011). Stratégies d'intégration professionnelle de femmes d'Afrique subsaharienne au Québec, [mémoire de maitrise], Université du Québec à Montréal, 146 p.

ORGANISATION INTERNATIONALE DUTRAVAIL (2016). « D'importantes disparités subsistent entre hommes et femmes sur les marchés du travail, selon l'OIT ", ONU info, réf. du 7 mars 2016, https://news.un.org/fr/story/2016/03/330642-dimportantes-disparites-subsistent-entrehommes-et-femmes-sur-les-marches-du

PICOT, Garnett, et Arthur SWEETMAN (2005). "Dégradation du bien-être économique des immigrants et causes possibles : mise à jour 2005 ", No 11F0019MIF au catalogue, № 262, Ottawa, Statistique Canada.

PIERRE, Myrlande (2005). « Les facteurs d'exclusion faisant obstacle à l'intégration socioéconomique de certains groupes de femmes immigrées au Québec : un état des lieux ", Nouvelles pratiques sociales, Vol. 17, $\mathrm{N}^{\circ}$ 2, p. 75-94.

SCHELLENBERG, Grant, et Hélène MAHEUX (2007). « Perspectives des immigrants sur leurs quatre premières années au Canada ", Tendances sociales canadiennes, № 11-008 au catalogue, 2-18, Ottawa, Statistique Canada.

YUVAL-DAVIS, Nira (2006). «Intersectionality and Feminist Politics », European Journal of Women's Studies, SAGE Publications, Vol. 13, No 3, p. 193-209, réf. du 30 mai 2018, https://hal.archivesouvertes.fr/hal-00571274/document 